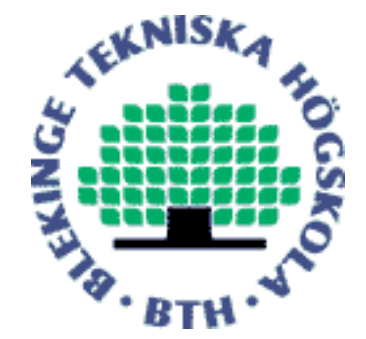

Copyright (C) 2010 IEEE.

Citation for the published paper:

A Structured Goal Based Measurement Framework Enabling Traceability and Prioritization

Tahir Touseef, Cigdem Gencel

6th Intern. Conf. on Emerging Technologies (ICET),

2010 Islamabad

This material is posted here with permission of the IEEE. Such permission of the IEEE does not in any way imply IEEE endorsement of any of BTH's products or services Internal or personal use of this material is permitted. However, permission to reprint/republish this material for advertising or promotional purposes or for creating new collective works for resale or redistribution must be obtained from the IEEE by sending a blank email message to pubs-permissions@iee.org.

By choosing to view this document, you agree to all provisions of the copyright laws protecting it. 


\section{A Structured Goal Based Measurement Framework Enabling Traceability and Prioritization}

\author{
Touseef Tahir \\ Department of Computer Science, \\ Comsats Institute of Information, Technology, \\ Lahore, Pakistan \\ touseeftahir@ciitlahore.edu.pk
}

\author{
Cigdem Gencel \\ Blekinge Institute of Technology, \\ SE-371 79 \\ Karlskrona, Sweden \\ cigdem.gencel@bth.se
}

\begin{abstract}
Software measurement has become a vital process in software organizations due its significant role in software management and software process improvement. A number of models and frameworks have been developed to support organizations to plan their measurement processes based on their goals. Goals Questions Metrics (GQM), Goal Question Indicator Model (GQIM) and Measurement Information Model (MIM) are among such models with various extensions. However, as the sizes of the software organizations and the number of stakeholders become larger, the measurement frameworks become more complex due to the high number of goals as well as the horizontal and vertical dependencies among them. These call for the need to have more visible and structured measurement process, which would enable prioritization and traceability. This paper discusses such a framework, called Structured Prioritized Goal Question Metrics (SPGQM) and a case study conducted in a CMMI Level 5 certified company in order to validate this framework.
\end{abstract}

Keywords-Goal Question Metric (GQM); software measurement program; software measurement process; prioritization; traceability.

\section{INTRODUCTION}

Software measurement programs help in both managing and implementing the software processes at different levels of the software organizations [1] as well as in enabling flow of data within the processes [2][3]. These give a competitive advantage to these organizations over the others who prefer traditional approaches [4].

Mendonca and Basili [5] defined the properties of a good measurement framework as being sound, complete, lean, and consistent. A number of models and approaches have been developed, which considerably helped the software organizations to initiate measurement programs with such characteristics. Some of the well-known ones include Goal Question Metric (GQM) paradigm [6][7], Goal Question Indicator Model (GQIM) [8], and Measurement Information Model (MIM)[9].

However, as the size of the organizations and the number of stakeholders become larger, the measurement frameworks become more complex [10] and difficult to manage due to the high number of goals, the horizontal and vertical dependencies among these goals [11], the links with the measures as well as the dependencies. In fact, current literature shows high failure rates in measurement programs [12][13] due to various reasons[14].

If the measurement framework is not structured well, this might lead to poor data use and loss of global understanding of the data [5]. Therefore, the high level goals and their relationships to the sub-goals of different stakeholders at the lowest levels of the organizations should be aligned by a more structured and visible way. Both horizontal and vertical dependencies among the goals, sub-goals and measures should be established.

Moreover, based on the constraints of an organization, changing priorities of stakeholders and their evolving goals, there should be a continuous optimization process for the measures selection.

This paper presents a framework, called SPGQM, which is an extension of GQM to address these requirements. We also present a case study we conducted in a CMMI Level 5 company to validate the defined framework. In Section II, the framework is presented. In Section III, the details of the case study and the results are discussed. In Section IV, the conclusions are provided.

\section{A STRUCTURED FRAMEWORK FOR GOAL BASED MEASUREMENT}

The main processes defined as part of SPGQM framework (see Figure 1) are discussed in the following paragraphs.

\section{A. Goals Definition Phase}

The goals are defined using a template to enable structured goals definition. In this framework, the goals and the subgoals of a software organization are defined in an iterative way. In [15], the purpose, issue, stakeholder's information need, scenario, and relevant entities are used to define measurement goals. The concept of "Information needs to track" [9] and goals categorization according the entity type (product, process and resources $[1,2]$ ) are also included in the template.

We made use of requirements engineering concepts [16] to make the goals traceable (just like for software requirements) and track information by including Goal Identifier, Version, 
Description, Author, Relevant Business goal, and primary measurement goal. The sub-goals of the primary goals are identified via the dependencies between goals. This is an iterative process. The same template is used for definition of primary measurement goals and sub goals.

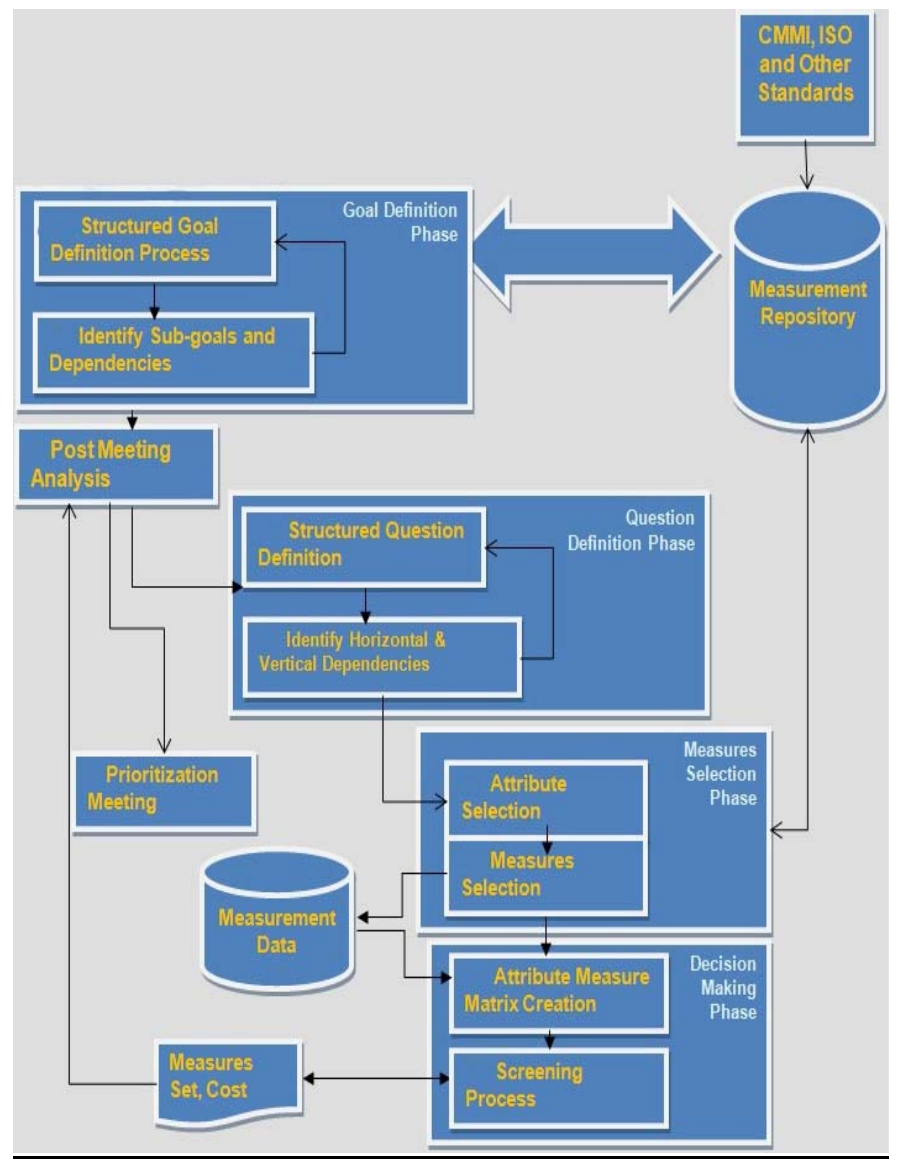

Figure 1 SPGQM Framework

Following are the descriptions of the attributes for structured goals definition template:

\section{1) Description}

The description contains general details of the goal that are required for the measurement program needed to be documented.

\section{2) Purpose}

It is an attribute of the goal which will describe the reason to study an object.

\section{3) Issue}

An issue is the property/quality of an object; i.e., project, process or resource to be studied. The issue is an important property which helps in identifying the metrics required to answer the achievement of goal.

\section{4) Category}

The goals are categorized according to the type of the software entity (see Item\#7) they are related to. The categories can be product, process or resource.

\section{5) Stakeholders for information need}

The stakeholders are any internal or external party. The internal stakeholders are the personnel who are associated with the measurement program or other parts of the organization. The external stakeholders can be customer, government agencies etc.

\section{6) Scenario/ Environment}

The scenario/context is the environment in which measurement is to be taken place. This attribute will also help in understanding the measurement process in the context of different phases of different software development life cycle.

\section{7) Relevant Entities}

The relevant entities are the objects, which are going to be analyzed for measurement. The relevant entities can be deliverables, work products, resources or processes in a software development life cycle.

\section{8) Information needs to track}

The concept of information needs is taken from measurement information model (MIM) [9]. The purpose of information need is to look for necessary information that is required to track the measurement goals.

\section{9) Relevant business goals}

The relevant business goals are provided with the measurement goals to analyze measurement goals according to the business goals. This also provides the traceability of the goals with respect to business goals. The literature shows that it's an effective approach to derive the measurement goals from business goals $[9,18]$.

\section{0) Primary measurement goals}

The structured goal definition provides the dependency and relationship between the primary measurement goal and its sub-measurement goals.

\section{1) Traceability information}

The traceability information is added to each goal so those modifications in the goals are recorded and the original version of the goal definition is also maintained to monitor the deviation in the goal definition and goal achievement. The traceability information includes identifier (unique ID), Author/resource, Description and Version.

Identifier: The purpose of the identifier is to allocate a unique value to each measurement goal and help in making the measurement goals traceable. The identifier property of the goals can contain the value so that it contains the project id/name and goal id e.g. itslearning-usability-01, where it's learning is project name and usability is the relevant goal.

Author/source: The author/source is a person in measurement program that has documented the measurement goal so that in future, if there is an ambiguity then it can be clarified. In most cases it might be a person relevant to measurement process.

Version: The purpose of the version attribute is to provide change management in measurement goals. There is a possibility that in long term measurement program that measurement goals are modified. Therefore, there is a need of 
a mechanism to track the changes and version attribute serve this purpose.

\section{B. Questions Definition Phase}

The questions relevant to each goal are defined in a structured way. The template for the structured question definition involves the following information:

\section{1) Associated Goal(s) identifier}

The question is identified according to the respective goal. If a question has a (vertical) dependency for more than one goal then this attribute should have the IDs of all the relevant measurement goals.

\section{2) Question identifier}

Each question has a unique identifier for traceability. This helps the measurement question to be uniquely identified in a measurement program.

\section{3) Issue}

The issue is the attribute that is going to be measured. The repetition of issue attribute in the question template is due to the reason that issue described in a goal can have more than one sub issues.

\section{4) Entity}

A specific entity/object whose attribute is going to be measured. A goal can have more than one entity but at the question level the specific entities that are needed to be studied are documented for entity attribute.

\section{5) Description}

The description field contains the overall description of the question.

\section{6) Stakeholders}

All the relevant stakeholders should be identified.

\section{7) Scenario/context}

The scenario/context is where the measurement is going to take place.

\section{8) Dependencies}

The horizontal dependencies between the questions are identified with the help of writing the unique identifiers of questions that are dependent. The horizontal dependencies are within the questions. The question $\mathrm{A}$ is horizontally dependent on question B, if the answer of a question B is to be used to complete the answer of question A.

There is a possibility that one question may be needed for more than one goal, such situation is called the vertical dependency between the goal and questions. The associated goal identifier attribute can contain more than one measurement goal IDs if there is more than one vertical dependency [11].

\section{Measures Selection and Decision Making}

This framework is developed as a continuation of our previous study in which we developed a model, called Optimum
Measures Set Decision Model (OMSD) [18] for deciding on the optimum set of measures.

OMSD is a heuristics based model also extending GQM, which aims to provide an optimum set of measures from a possible large finite set of measures.

During the decision making for the selection of measures, OMSD takes controlled inputs in the form of constraints (time and cost limits of the organization) and other factors such as the repetition and priority of the measures. The constraints act as thresholds that are utilized as process terminators.

In this model, only the lowest level measurement goals were taken into account when determining constraints and the 'priority' value of a measure was given a-priori as if the value is inherited from the priority of the business goals and related measurement goals.

It not only helps in identification and selection of measures to answer the relevant questions but it also provides the optimization mechanism of the measures. The OMSD model has five main steps:

- Selection of category of a question (Process, Product, Resources)

- Identification of the attributes to be measured in the questions.

- $\quad$ Selection of measures from the measurement pool or using the measures defined in software engineering standards.

- The development of factor and attribute tables with the help of data collection.

- Decision making by using heuristic approach to select optimum set of measures

\section{Cost benefit analysis}

It is an informal approach for decision-making. In this framework, OMSD model is used as a basis for it. Each goal has a set of question and measures. The OMSD model is used to calculate the cost of optimum set of measures. The cost of measures for a goal is used in the analysis against the overall benefits of the goal. It helps the measurement responsible in the prioritization of the goal. This approach aids in making the prioritization on the basis of cost and frequency, importance, usage of measures (identified in OMSD) against benefits of goals which make task of goal prioritization less error prone. In SPGQM, the 100 dollar method [16] is used to assign priority values to the goals. In this method each stakeholder (internal and/or external) distributes 100 points among goals according to their importance and cost. The goals with the higher value have the higher priority. At the end, mean of the values (given to the goals by all the participants) are calculated [11].

\section{CASE STUDY}

We conducted a case study in a CMMI Level 5 software company ' $A$ ' to evaluate the proposed framework (see Figure 2). 


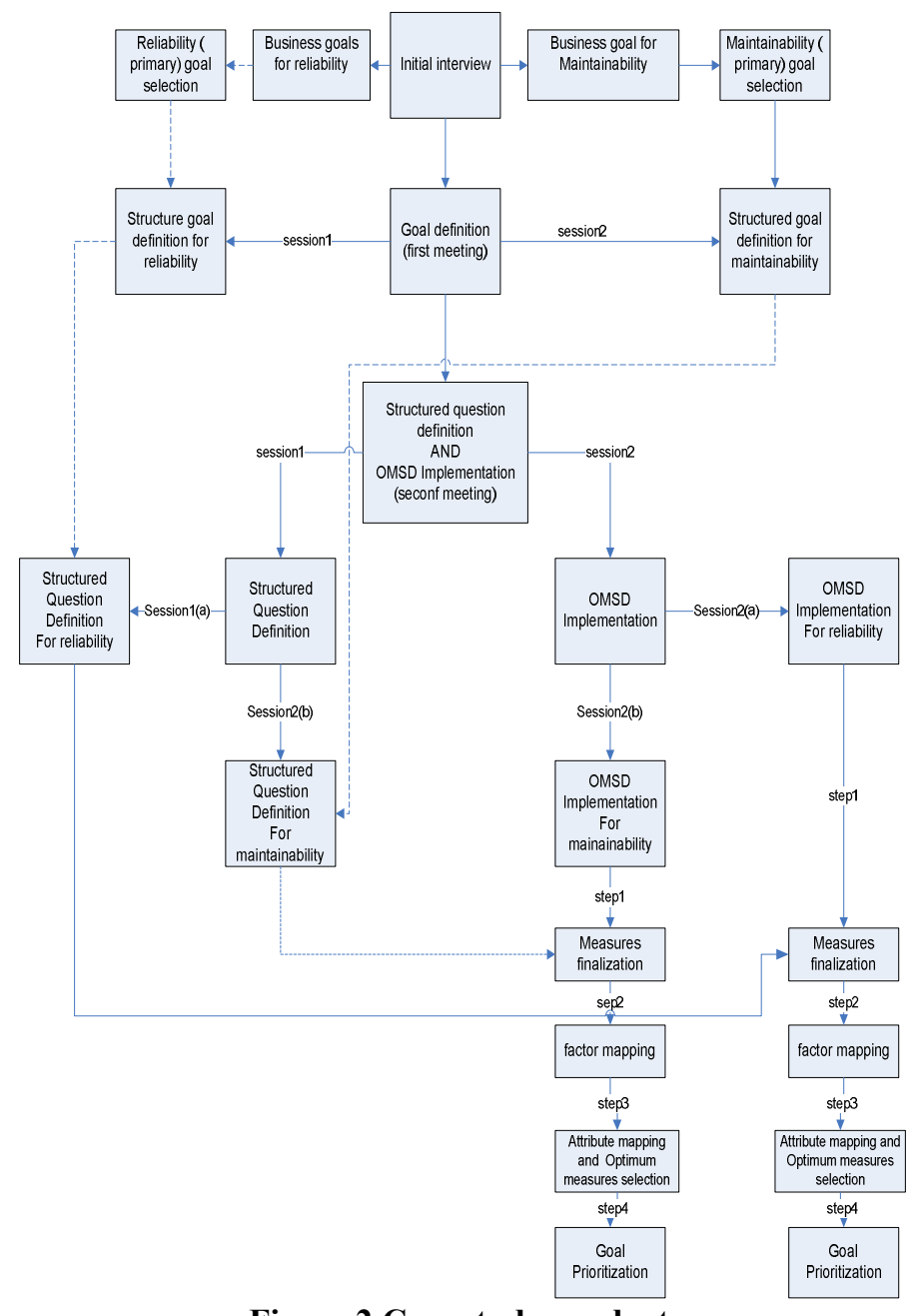

Figure 2 Case study conduct

It is an IT-based company established in 1987 in USA, which provides IT solutions and services around the globe. Main IT solutions involve warehousing, software development, modification and maintenance. The company provides telecommunication, human resource management and financial management services.

We conducted an interview with the project manager first to get insight about the organization. Our interview had three parts; i.e. interviewee introduction, organization introduction and measurement processes. In the organizational introduction part, we received information about the types of projects, software development life cycle used, software standards and models. In the measurement processes, we received information about measurement programs, common set of software measures and how these measures are collected, stored and analyzed.

Two projects (e-banking and e-management system) were selected as the case projects at the end of the interview. We reviewed the Software Requirement Specifications (SRS) for these projects before the implementation of SPGQM. After analyzing the SRS and the information received during the interviews, we selected the primary measurement goals as reliability and maintainability improvement for the case projects. The selection of only one primary measurement goal from each project was due to limited time available for the case study.

We sent the executive summary to the case company before conducting the case study. The executive summary included the details of SPGQM and the plan of the case study. We also showed some examples of how to define the goals and the question. An example about OMSD application was also provided to the company.

The participants of the case study were the project manager, business analyst and the technical team lead. The case study conduct is discussed in the following paragraphs according to the main processes of SPGQM.

\section{A. Structured Goals Definition}

In the first meeting, the implementation started with the goals definition by filling in the templates of the goals using Google docs. The meeting was divided into two sessions. Each part consisted 45 minutes to define the sub-goals for a primary goal of each project. Three sub goals for each primary goal were finalized for each primary measurement goal during the structured goal definition sessions. Figure 3 shows the results oft the iterative goals definition session.

The second meeting was held in two main sessions: structured questions definition and measures selection.

\section{B. Structured Questions Definition}

The questions for the goals of each project were defined using the SPGQM question definition template. Two structured question definition sessions were held for this purpose. Each session was 45 minutes long. The finalization of the question was on the basis of agreement on inclusion of question by the manager and at least one of the business analyst/technical team lead. Figure 4 shows the results of the structured questions definition session for the measurement goals.
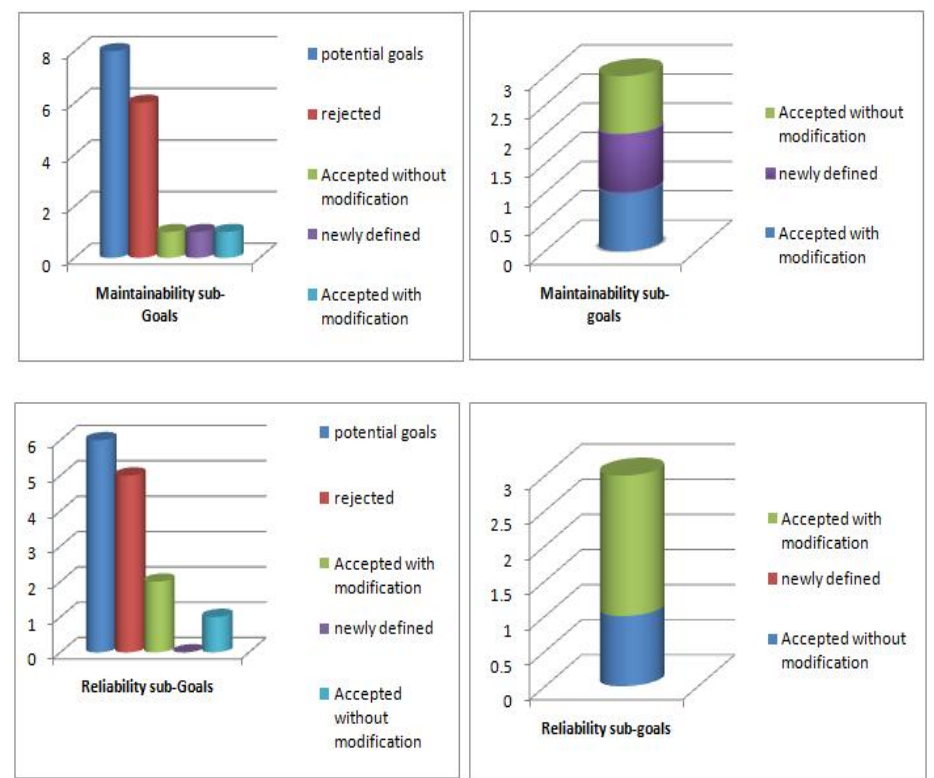

Figure 3 Results of the Iterative Goal Definition Session 


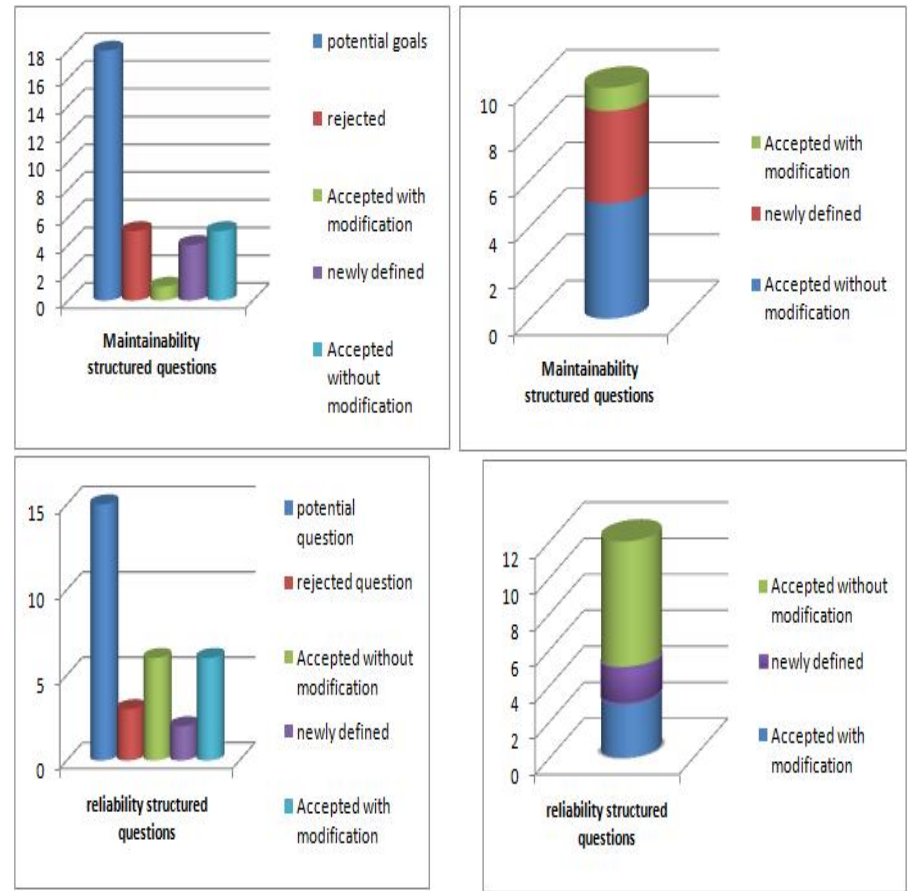

Figure 4 Results of the Question Definition Session

\section{Measures Selection and Decision Making}

This phase involved two sub sessions. In the first sub session, the candidate measures from the available software engineering standards were identified. Then, for each project the set of selected measures for each goal were mapped on to a factor table [31].

Later on, the attribute mapping [31] was done for the measures to identify the attributes for each measure that can help in decision making on the basis of available budget, importance and usage of the measure.

The participants from the company were given different roles for the prioritization task. The project manager was asked to prioritize the goals from the user perspective. The technical team lead was asked from the organization/technical perspective and the business analyst on a general level. The average of all the priorities for each sub-goal was taken at the end to have the final value of priorities for each sub goal.

Then, for each project OMSD was used for the final decision making on the basis of available budget, importance and usage frequency of the measures.

\section{CONCLUSION}

This paper introduces a new structured goal based measurement framework for better planning of measurement programs.

SPGQM enables the idea of traceability, prioritization and reusability with the help of structured and visible definition and association of the goals, questions and measures. With the integration of the OMSD model, this framework also helps in deciding on the selection of optimum set of measures based on the priority of the goals as well as other constraints.
As a future work, we plan to automate SPGQM framework and incorporate new techniques for prioritization, optimization and decision-making.

\section{REFERENCES}

[1] M. Berry and M. F. Vandenbroek, "A Targeted Assessment of the Software Measurement Process," in Proceedings of the 7th International Symposium on Software Metrics (ISSM '01), Washington, DC, IEEE Computer Society, pp. 222-235, 2001.J. Clerk Maxwell, A Treatise on Electricity and Magnetism, 3rd ed., vol. 2. Oxford: Clarendon, 1892, pp.68-73.

[2] C.Karner and W.P.Bond,"Software Engineering: What do they measure and how do we know?," In proceeding of the 10th international metrcis symposium, IEEE computer society,pp.2-5,2004.

[3] D. B. Simmons, "A win-win metric based software management approach," IEEE Transactions on Engineering Management, vol. 39, Texas: IEEE, pp. 32-41, 1992.

[4] F. Niessink and H. V. Vliet, "Towards Mature Measurement Programs," In Proceedings of the 2nd Euromicro Conference on Software Maintenance and Reengineering (CSMR'98), March 08 - 11, IEEE Computer Society Washington, DC, USA, pp.82. 1998.

[5] M. G. Mendonça and V.R. Basili, "Validation of an Approach for Improving Existing Measurement Frameworks", IEEE Transactions on Software Engineering, 26, 6, pp. 484-500, June 2000.

[6] Basili, V.R, and Weiss, D.M.: A Methodology for Collecting Valid Software Engineering Data. IEEE Transactions on Software Engineering, Vol.SE-10, no.6, pp. $728--738$ (1984)

[7] Basili, V.R, and Rombach, H.D.: The TAME Project: Towards Improvement-Oriented Software Environments. IEEE Transactions on Software Engineering. SE-14 No.6, pp. 758--773 (1988)

[8] R. E. Park, W. B. Goethert and W. A. Florac, Goal-Driven Software Measurement - A Guidebook, Handbook CMU/SEI-96-HB-002, Software Engineering Institute, Carnegie Mellon University, Hanscom, MA, 1996.

[9] ISO/IEC 15939 International Standard: Software engineering - Software measurement process. Ref Number ISO/IEC 15939:2002(E), 2002.

[10] Ardimento, P., Baldassarre, M.T., Caivano, D., Visaggio, G.:Multiview Framework for Goal Oriented Measurement Plan Design, F. Bomarius and H. Iida (Eds.): PROFES 2004, LNCS 3009, Springer-Verlag Berlin Heidelberg, pp. 159-173, 2004.

[11] P. Berander, and P. Johnsson, "A goal question metric based approach for efficient measurement framework definition," In Proceedings of the $2006 \mathrm{ACM} / \mathrm{IEEE}$ international Symposium on Empirical Software Engineering (ISESE '06), September 21 - 22, Rio de Janeiro, Brazil, ACM: New York, NY, 316-325. 2006.

[12] Bundschuh, M., and Dekkers, C.: The Measurement Compendium: Estimating and Benchmarking Success with Functional Size Measurement. Springer-Verlag, Berlin Heidelberg (2008)

[13] Goethert, W., Hayes, W.: Experiences in Implementing Measurement Programs. Technical Note, Software Engineering Institute, Carnegie Mellon University, CMU/SEI-2001-TN-026 (2001)

[14] Gopal, A., Krishnan, M.S, Mukhopadhyay, T., Goldenson, D.R.: Measurement Programs in Software Development: Determinants of Success. IEEE TSE, Vol.28, Issue 9, pp. 863-875, 2002.

[15] H. Koziolek, "Dependability Metrics", vol. 4909, Chapter: Goal Question Metric, Springer-Verlag Berlin Heidelberg, Pp. 39-42, 2008.

[16] I. Sommerville, G. Kotonya; Requirement Engineering: Process and Techniques, United Kingdom: 1998.

[17] J. Gorbutt and P. Layzell, "Extending the AMI approach to encompass the Foundation activity in Software Technology and Engineering Practice," In Proceedings of Eighth IEEE International Workshop on Incorporating Computer Aided Software Engineering (IWICASE '97), July 14-18, London UK: IEEE, Pp. 198-208, 1997.

[18] A. M. Bhatti, H. M. Abdullah, and C. Gencel, "A model for selectng an Optimum Set of Measure in Software Organizations," in Proc. EurSPI, 2009. 